\title{
Deep perifoveal vessel density as an indicator of capillary loss in high myopia
}

\author{
Dan Cheng ${ }^{1} \cdot$ Qi Chen ${ }^{1} \cdot$ Yufei $\mathrm{Wu}^{1} \cdot$ Xueting $\mathrm{Yu}^{1} \cdot$ Meixiao Shen ${ }^{1} \cdot$ Xiran Zhuang $^{1} \cdot$ Zhongxu Tian $^{1} \cdot$ Ye Yang ${ }^{1} \cdot$ \\ Jianhua Wang ${ }^{2} \cdot$ Fan Lu $^{1} \cdot$ Lijun Shen $^{1}$
}

Received: 8 May 2018 / Revised: 22 May 2019 / Accepted: 15 August 2019 / Published online: 16 September 2019

(c) The Author(s), under exclusive licence to The Royal College of Ophthalmologists 2019

\begin{abstract}
Objectives This study investigates the macular vasculature of both vascular layers in different degrees of myopia by optical coherence tomography angiography (OCTA).

Methods One hundred and forty-five eyes of 145 healthy subjects with spherical equivalents (SE) ranging from +0.50 to -16.50 dioptres were divided into three groups. The foveal avascular zone (FAZ) area and vessel density (VD) of OCTA images were compared.

Results Compared with other groups, high myopia had a larger FAZ and less perifoveal VD in both layers and less deep total VD. Compared with other myopia, high myopia had less total VD in the superficial layer and parafoveal VD in the deep layer. Between emmetropia and low myopia, there was a difference only in the deep parafoveal VD. Except for the parafoveal VD in both layers and the superficial total VD, there were significant correlations of the FAZ with axial length (AL) and SE as well as VD. The deep perifoveal VD was most associated with high myopia.

Conclusions An increased FAZ and decreased VD in both layers were correlated with AL elongation. The decreasing deep perifoveal VD was most associated with high myopia. OCTA may provide additional information regarding the progression of pathologies in high myopia.
\end{abstract}

\section{Introduction}

Myopia is prevalent in most populations, particularly in Asian countries such as China. Complications related to myopia, such as glaucoma, maculopathy, retinal detachment, and myopic macular degeneration, are major causes of visual dysfunction and blindness [1,2]. Previous histopathologic studies have shown that changes in the retinal macular vasculature, including thinning of the vascular layer, capillary nonperfusion, ischemia, and hypoxia, are associated with the development of refractive errors or axial

These authors contributed equally: Dan Cheng, Qi Chen

Lijun Shen

ljshenysg@163.com

1 School of Ophthalmology and Optometry, Wenzhou Medical University, Wenzhou, Zhejiang, China

2 Department of Ophthalmology, Bascom Palmer Eye Institute, University of Miami, Miami, FL, USA length (AL), which are considered to increase the susceptibility to pathologic myopia, such as glaucoma and maculopathy [3-5].

Based on previous evidence, changes in retinal vasculature have been extensively studied in myopia, using several clinical imaging methodologies to identify early indicators of vascular changes [10-16], but the findings remain controversial. Decreased retinal vessel diameter, increased foveal avascular zone (FAZ), and lack of changes of these vascular parameters have all been found in myopic eyes [6-11] and animal models [12]. Several reasons may contribute to the discrepancies in previous studies. One is the methodologies. Most of the previous studies used colour fundus photographs or fluorescein angiography (FA) [5, 13] to evaluate the retinal vasculature. However, these imaging modalities are either invasive techniques or cannot image the macular microvascular network and thus cannot detect the subtle changes at the very early stage in myopia. The other is the correction of the magnification induced by the AL. Some studies did not consider the correction of the lateral scale due the different magnification in myopia with the refractive error or AL increase, which could affect the 
measurement of the retinal vascular parameters. Considering the changes in the macular vascular network may be an early indicator to identify myopia at risk of developing pathology; more work is necessary to characterize the association of the changes in macular vasculature with myopia by using more accurate and higher resolution methods.

Optical coherence tomography angiography (OCTA) is a recent, rapid, non-invasive technology that provides highresolution retinal capillary detail for differential layer analysis [13, 14]. Previous studies have demonstrated that OCTA offers results with high intra-visit repeatability, and inter-visit reproducibility $[15,16]$. The purpose of this study is to investigate the association of the changes in the macular vasculature in different vascular layers with myopia, by OCTA, and identify the indicator of macular microvascular network loss in myopia without evidence of clinically overt retinal disease, based on the quantitative parameters.

\section{Methods}

\section{Subjects}

One hundred forty-five volunteers were recruited from January 2016 to April 2017. Each subject underwent a complete ocular examination, including the following: bestcorrected visual acuity (BCVA); slit-lamp biomicroscopy; subjective refraction for obtaining the spherical equivalent (SE); and IOL Master (Carl Zeiss Meditec, Jena, Germany) for obtaining the AL, intraocular pressure measurement, and funduscopy. This project was approved by the Ethics Committee of the eye hospital of Wenzhou Medical University and was performed in accordance with the tenets of the Declaration of Helsinki. Informed consent was signed by all the subjects.

The enroled subjects were divided into the following three subgroups according to SE: emmetropia (from $+0.50 \mathrm{D}$ to $-0.50 \mathrm{D}, n=30$ ), low-to-moderate myopia (from $-0.50 \mathrm{D}$ to $-6.00 \mathrm{D}, n=64)$, and high myopia $(<-6.00 \mathrm{D}, n=51)$. Inclusion criteria were as follows: BCVA of $16 / 20$ or better, astigmatism lower than $2.00 \mathrm{D}$, and less than $2.00 \mathrm{D}$ difference between the two eyes. Exclusion criteria were ocular surgery or trauma; IOP $>21 \mathrm{~mm} \mathrm{Hg}$; myopic macular degeneration; a history of vitreoretinal disease or systemic disease that could affect the eyes, such as diabetes mellitus or hypertension.

\section{OCTA data acquisition and processing}

OCTA scans were obtained with the spectral-domain system RTVue-XR Avanti (Optovue, Inc., Fremont, CA,
USA). Orthogonal registration and merging of two consecutive scans were used to obtain OCTA volume scans of a central $3 \times 3 \mathrm{~mm}$ area of the right eye for each subject. Using the automated software algorithm, OCTA images of the superficial and deep capillary networks were generated separately. The superficial network extends from $3 \mu \mathrm{m}$ below the internal limiting membrane to $15 \mu \mathrm{m}$ below the inner plexiform layer (IPL) according to the automated setting. The deep capillary network extends from 15 to $70 \mu \mathrm{m}$ below the IPL [15]. Custom-built imaging software implemented in MATLAB (Natick, MA, USA) was used to separate the foveal capillary vascular ring from the background image by using an active contour model based on the level-set method [17] and automatically measure the FAZ area in both superficial and deep network (Fig. 1a-c, $\mathrm{e}-\mathrm{g}$ ). Vessel density (VD), calculated as the percentage of pixels with flow signal greater than the threshold, was also automatically measured. Before magnification correction, in both superficial and deep layers, total VD was calculated for the ring-shaped area between a 0.5 and $1.25 \mathrm{~mm}$ radius from the centre of the macula. Parafoveal and perifoveal VD were calculated for the area between a 0.5 to $0.875 \mathrm{~mm}$ and 0.875 to $1.25 \mathrm{~mm}$ radius from the foveal centre, respectively (Fig. 1d, h). To reduce the effects of decentration of the FAZ and the artefacts at the edge of the scan, a circular area with a diameter of $2.5 \mathrm{~mm}$ was used instead of the entire $3 \times 3 \mathrm{~mm}^{2}$ area.

The magnification of the retinal images obtained by the optic devices is affected by AL and SE [18-20]. To eliminate the effect of magnification, images obtained from OCTA were corrected using the AL by Bennett's formula $[19,21,22]$.) The relationship between the OCT image measurements and the actual scan diameter was expressed by the formula $t=p \times q \times s$, where $t$ represented the actual AL, $p$ represented the magnification factor determined by the camera of the OCT imaging system, $q$ represented the magnification factor in relation to the eye, and $s$ represented the original measurement value obtained from the OCT image. The correction factor $q$ was determined with the equation $q=0.01306 \times(\mathrm{AL}-$ 1.82) [21]. For example, we use the RTVue-XR Avanti OCT to image a highly myopic eye with an AL of $29.45 \mathrm{~mm}$, which is the longest AL in our study. Before magnification correction, in the superficial layer, the FAZ area is $0.316 \mathrm{~mm}^{2}$. The actual scanning area in this eye would be $\left[(29.45-1.82)^{2} /(24.46-1.82)^{2}\right] \times$ $0.316=0.471 \mathrm{~mm}^{2}$. For VD calculation, after magnification correction, parafoveal and perifoveal VD were calculated for the ring-shaped area between a 0.5/ $[[(29.45-1.82) /(24.46-1.82)]=0.492 \mathrm{~mm}$ to $0.875 /$ $[[(29.45-1.82) /(24.46-1.82)]=0.758 \mathrm{~mm}$ and 0.758 to $1.25 /[[(29.45-1.82) /(24.46-1.82)]=1.024 \mathrm{~mm} \quad$ radius from the centre of the macula, respectively. 


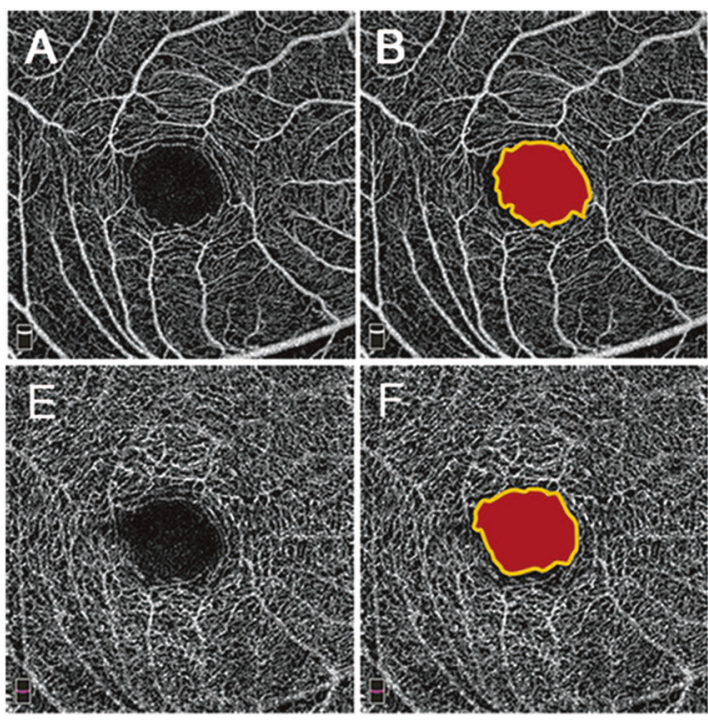

Fig. 1 Representative optical coherence tomography angiography (OCTA) and post-processed images illustrating the automated analysis algorithm before magnification correction. An original OCTA image in the superficial (a) and deep (e) retinal layers from a high myopic eye, respectively; b, f The foveal avascular zone (FAZ) was separated from the background image and the area was automatically measured;

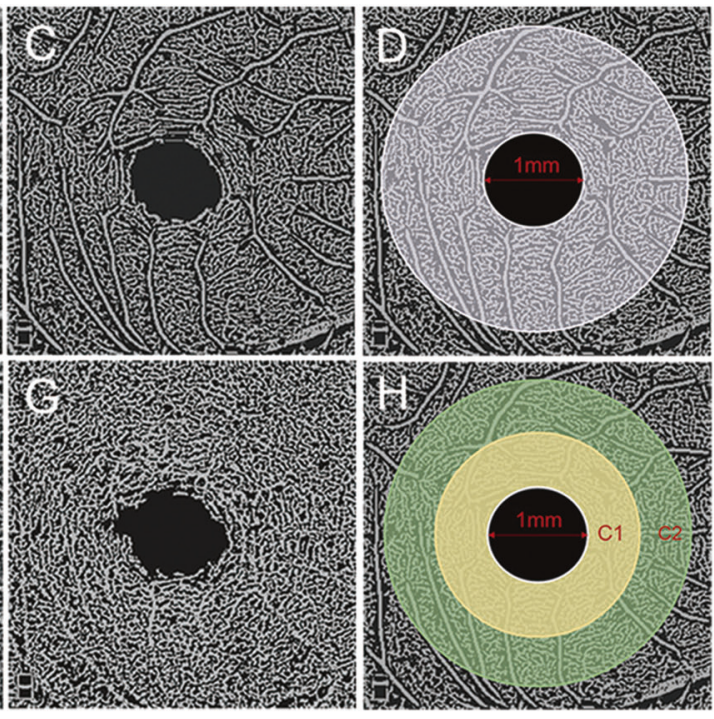

a skeletonized image in the superficial (c) and deep (g) retinal layers, respectively. These images were used for vessel density(VD) analysis. d Total VD was calculated for the ring-shaped area between a 0.5 and $1.25 \mathrm{~mm}$ radius from the fovea centre. $\mathbf{h ~} \mathrm{C} 1$ and $\mathrm{C} 2$ were $0.5-0.875$ and $0.875-1.25 \mathrm{~mm}$ radius ring-shaped areas from the fovea centre, respectively
Table 1 Subject characteristics of emmetropic, low-to-moderate myopic, and high myopic groups

\begin{tabular}{llllr}
\hline & Emmetropia & Low-to-moderate myopia & High myopia & $P$ Value \\
\hline No. subjects & 30 & 64 & 41 & \\
Age, years; range & $26.87 \pm 4.67 ; 20-36$ & $25.11 \pm 3.70 ; 21-41$ & $25.27 \pm 4.92 ; 16-42$ & $0.170^{\mathrm{b}}$ \\
Sex, male:female & $13: 17$ & $25: 39$ & $20: 31$ & $0.916^{\mathrm{a}}$ \\
Al, mm; range & $23.42 \pm 0.69 ;$ & $24.98 \pm 0.92 ; 23.06-26.60$ & $26.82 \pm 1.14 ;$ & $<0.001^{\mathrm{b}}$ \\
& $22.11-24.61$ & & $24.35-29.45$ & \\
SE, D; range & $-0.09 \pm 0.30 ;-0.50$ & $-3.42 \pm 1.45 ;-5.75$ & $-8.71 \pm 2.01 ;-16.50$ & $<0.001^{\mathrm{b}}$ \\
& to +0.50 & to -0.75 & to -6.00 & \\
\hline
\end{tabular}

$A L$ axial length, $S E$ spherical equivalent, $D$ dioptre

${ }^{a}$ For sex comparisons, the Pearson chi-square was performed as appropriate

${ }^{b}$ For three-group comparisons, the analysis of variance or Kruskal-Wallis tests were performed as appropriate

\section{Statistical analysis}

All data were expressed as means \pm standard deviations and were analyzed with SPSS software (version 22.0, SPSS Inc., Chicago, IL, USA). Categorical variables were compared between groups by chi-square or Fisher exact tests. The Kolmogorov-Smirnov test was used to verify distribution normality for continuous variables. One-way analysis of variance was performed for comparing continuous variables among three groups and least significant difference was used for multiple comparisons. Associations among intra-retinal FAZ area, VD, AL, and SE were determined with Pearson correlation tests. Logistic regression was used to determine the odds ratio (OR) for high myopia in relation to variations in FAZ and VDs in both retinal layers. $P$-values $<0.05$ indicated statistical significance.

\section{Results}

\section{Demographics}

A total of 145 eyes from 145 subjects with mean SEs of $-4.59 \pm 3.68 \mathrm{D}$ (ranging from -16.50 to $+0.50 \mathrm{D}$ ) and mean ALs of $25.31 \pm 1.59 \mathrm{~mm}$ (ranging from 22.11 to $29.45 \mathrm{~mm}$ ) were enroled in this study. According to the SE, they were divided into three groups, whose characteristics are detailed in Table 1. There were no significant differences in age $(P=0.170)$ or sex $(P=0.916)$ among the three groups. 


\section{Intergroup differences: FAZ and VD in the superficial and deep vascular layers}

The FAZ area, parafoveal, perifoveal, and total VD in both vascular layers were determined before and after correction for ocular magnification for all the OCTA images obtained from each subject. Before the correction for ocular magnification, there were significant differences in the perifoveal VD in both vascular layers $(P=0.012$ and 0.018 for superficial and deep layer, respectively). Although there was no statistical significance, in both layers, the mean value of the FAZ was even smaller in both myopic groups compared with the emmetropia group.

After the correction for ocular magnification, among the three groups, there were significant differences of the FAZ area $(P=0.002$ and $<0.001$ for superficial and deep layer, respectively) and perifoveal VD $(P<0.001)$ in both vascular layers, and parafoveal $(P=0.025)$ and total VD $(P=0.001)$ in the deep layer. Compared with the other two groups, high myopia had a larger FAZ area and less perifoveal VD in both layers and less total VD in the deep layer (Fig. 2). In addition, compared with low-to-moderate myopia, high myopia had less total VD in the superficial layer and parafoveal VD in the deep layer $(P<0.05)$. Between emmetropia and lowto-moderate myopia groups, there was difference only in the parafoveal VD of the deep layer $(P<0.05)$.

\section{Correlations of vascular parameters with AL and SEs}

After magnification correction, except for the parafoveal VD in both layers and total VD in the superficial layer, there were significant correlations of the FAZ area with $\mathrm{AL}$ and
SE as well as VD (Table 2). The FAZ area in the superficial and deep layers was positively correlated with AL (Pearson $r=0.216$ and 0.467 , respectively; $P<0.01$ ) and negatively correlated with SE (Pearson $r=-0.260$ and -0.427 , respectively; $P<0.01)$. In the superficial layer, less perifoveal VD was correlated with longer $\mathrm{AL}(r=-0.27, P=$ $0.001)$ and higher SE $(r=-0.294, P<0.001)$. Total VD was positively correlated with SE $(r=0.219, P=0.008)$. In the deep layer, less perifoveal and total VD correlated with longer $\mathrm{AL}(r=-0.505$ and $-0.32, P<0.001)$ and higher SE $(r=0.527$ and $0.37, P<0.001)$.

Table 2 Correlations of foveal avascular zone and vessel density with axial length and spherical equivalent

\begin{tabular}{lccccc}
\hline & \multicolumn{2}{c}{ Axial length } & & \multicolumn{2}{c}{ Spherical equivalent } \\
\cline { 2 - 3 } & $r$ & & & $r$ & $P$ \\
\hline Superficial FAZ & & & & \\
FAZ area & 0.216 & $0.009^{\mathrm{a}}$ & & -0.26 & $0.002^{\mathrm{a}}$ \\
Parafoveal VD & 0.068 & 0.417 & 0.011 & 0.895 \\
Perifoveal VD & -0.27 & $0.001^{\mathrm{a}}$ & 0.294 & $<0.001^{\mathrm{a}}$ \\
Total VD & -0.157 & 0.06 & & 0.219 & $0.008^{\mathrm{a}}$ \\
Deep FAZ & & & & \\
FAZ area & 0.467 & $<0.001^{\mathrm{a}}$ & -0.427 & $<0.001^{\mathrm{a}}$ \\
Parafoveal VD & 0.062 & 0.456 & 0.013 & 0.874 \\
Perifoveal VD & -0.505 & $<0.001^{\mathrm{a}}$ & 0.527 & $<0.001^{\mathrm{a}}$ \\
Total VD & -0.32 & $<0.001^{\mathrm{a}}$ & 0.37 & $<0.001^{\mathrm{a}}$ \\
\hline
\end{tabular}

" $r$ " is Pearson correlation co-efficient. " $P$ " is $P$ value

$F A Z$ foveal avascular zone, $V D$ vessel density

${ }^{\text {a }}$ Significant correlation with axial length and spherical equivalent after correction for ocular magnification
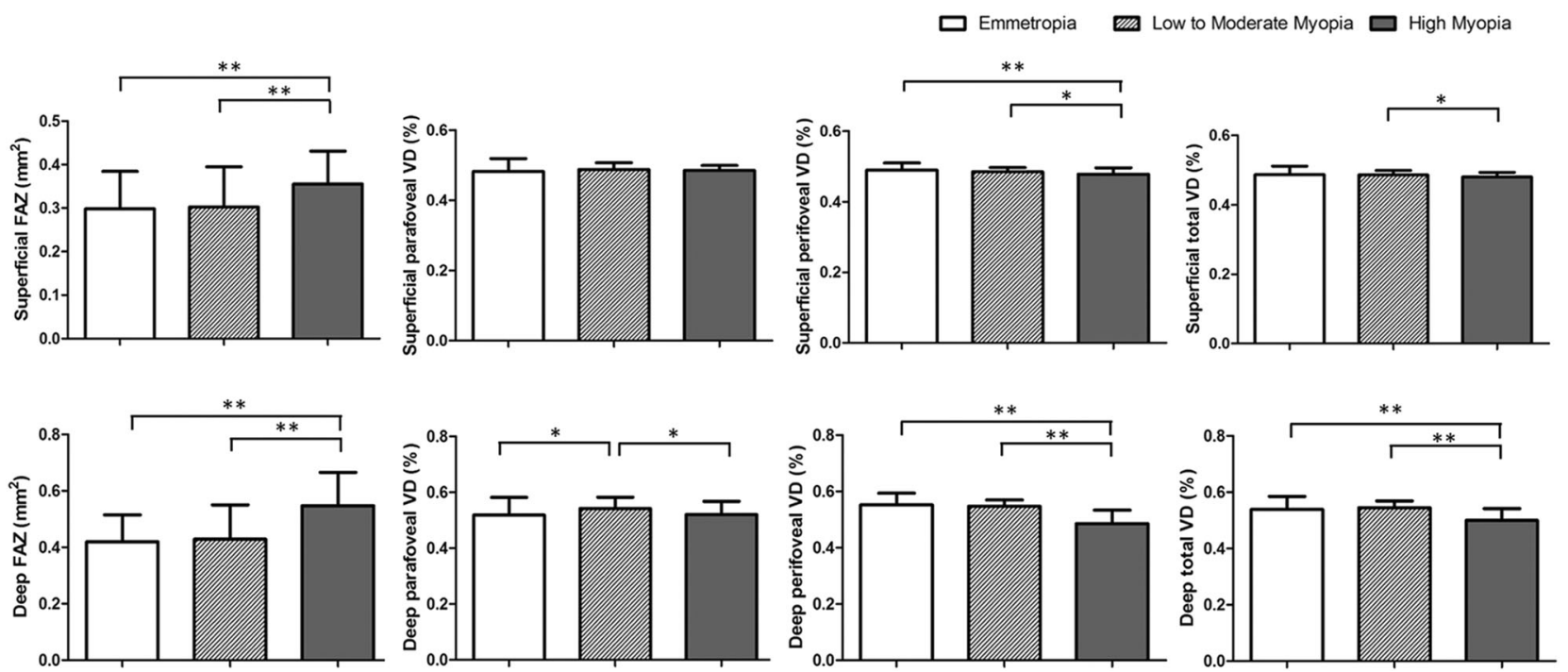

Fig. 2 FAZ and vessel density (VD) in the superficial (top) and deep (bottom) vascular layers among three groups. FAZ foveal avascular zone, VD vessel density. $* P<0.05, * * P \leq 0.01$ 
Relationship between vascular parameters and high myopia

We performed logistic regression to analyse the OR and determine the relationship between parameters of vascular morphology in both vascular layers and high myopia. After adjustments for age and sex, in both layers, high perifoveal VD (OR 0.74 [95\% CI: 0.58-0.94], $P=0.013$ and 0.67 [0.59-0.78], $P<0.001$ for superficial and deep layer, respectively) and total VD (0.79 [0.63-0.99], $P=0.040$ and 0.73 [0.65-0.83], $P<0.001)$ were identified as independent predictive, protective factors for high myopia. A large FAZ (OR 1.10 [95\% CI: 1.05-1.15] and 1.10 [1.06-1.15] for superficial and deep layer, respectively, $P<0.001)$ was considered to be a risk factor.

\section{Discussion}

As a risk factor for complications related to high myopia, changes in retinal vasculature have been studied widely to clarify the mechanism of development of myopic complications. However, the precise nature is still being debated. The major reasons might be attributed to the difficulties in accurately assessing the capillary network of the retina and the different methods being used. Using quantitative parameters of retinal microvascular morphology based on OCTA images, the present study assessed the capillary loss in myopic patients. The major finding is that the FAZ increased in high myopia, and total and perifoveal VD decreased. Furthermore, these changes are associated with the AL elongation. Notably, perifoveal VD in the deep layer could indicate the need to detect capillary loss in high myopia with more sensitivity.

Our finding is in line with previous studies by various methods [6, 7, 23, 24]. By analysis of colour Doppler imaging, Dimitrova et al. [23] found that retinal ciliary blood velocity decreased with the increase of the degree of myopia. Using laser Doppler velocimetry, Shimada [6] reported that retinal blood flow was decreased in high myopia, mainly due to the narrowing of the retinal vessel diameter. By Heidelberg retinal flowmeter, Man et al. suggested that longer eyes have decreased retinal function and $\mathrm{O}_{2}$ consumption, which was in accordance with the retinal capillary loss in high myopia [24]. Later, La Spina et al. [7] also demonstrated the reduced diameter of retinal vessels in high myopia by dynamic vessel analyser. This early macular microvessel damage is hypothesized to result from the mechanical expansion induced by the excessive elongation of the eyeball in high myopia [6], because longer eye might result in the straightening and decreased diameter of the retinal vessels. Another explanation might be the reduction in retinal blood flow induced by the decrease in metabolic demands associated with axial elongation [24]. Because retinal blood flow is directly correlated with the amount of arterial and venular $\mathrm{SO}_{2}$, the capillary loss may be due to this reduction in retinal blood flow. With the development of OCTA, Yang et al. [25] revealed retinal microvascular alterations in high myopia, which correlated with AL elongation. Li et al. [26] and Al-Sheikh et al. [27] also reported the decreased VD in high myopia subjects based on quantification of OCTA images. However, these studies only recruited patients with high myopia with an SE higher than $-6 \mathrm{D}$. The current study enroled 145 subjects, including 64 with low-to-moderate myopia and 51 with high myopia. The result demonstrated limited vascular alterations between emmetropia and low-to-moderate myopia. Furthermore, such a sample size with continuous variation of SEs may provide a reliable analysis of correlation between vascular parameters with $\mathrm{AL}$ and SE.

Interestingly, in our study, among all the vascular parameters in both layers, perifoveal VD in the deep layer was most associated with retinal capillary loss related to myopia. Previous studies have demonstrated that the superficial and deep capillaries contributed to different segments of retinal metabolism under normal conditions. Unlike the superficial layer, the deep capillary plexus contributes to part of the photoreceptor inner segment oxygen requirements (10-15\%) [28]. More important, the inner retinal vascular contribution to the metabolic needs of the outer retina became more significant in cases of choroidal vasculature dysfunction [29]. Thus, along with the choroidal disruption during the progression of high myopia [27], the correlation between the deep capillary and the outer retinal layers might become gradually more significant. In high myopia, the disorganization and thinning of the outer retina mainly due to ocular elongation, especially in the pericentral and peripheral retina, has been widely reported [30, 31]. This outer retinal disruption may decrease the need for oxygen of photoreceptor segments and further decrease the deep capillary circulation [23]. Further, to preserve the central retina, reduction of the vasculature may occur in the perifoveal region prior to the centre [30]. Investigation of the perifoveal VD in the deep layer may be useful and sensitive in evaluating the retinal vasculature of myopia. Because myopia-related complications are associated with abnormalities of retinal vessels $[4,5,8,32]$, a longer $\mathrm{AL}$ and reduced microcirculation was suggested as contributing factors to the development of damage from complications $[8,33,34]$. Identifying patients with capillary loss before or during a pathologic stage may help clinicians apply preventive procedures and potentially be useful in predicting response to therapy.

In this study, increases in the FAZ area and decreases in retinal vessels were correlated with the longer $\mathrm{AL}$ and higher dioptre of myopia. Among all the correlations, the 
perifoveal VD in the deep layer was the most significant, indicating that deep perifoveal VD is a useful indicator of retinal vasculature dysfunction with longer eyes. Our result was consistent with Patton et al. [35], who suggested that narrowed retinal vessel diameters were associated with increased AL, indicating the altered retinal blood flow seen in myopia. Likewise, both adult and children studies have shown a significant narrowing of retinal vessel calibre with increasing AL [34, 36, 37]. On the other hand, Yu et al. [16] and Tan et al. [11] reported that SE and AL did not significantly affect the FAZ area. However, the refractive error span of subjects in their study was too limited to study such correlation. In our study, regarding ocular magnification, we included subjects with SEs from -16.50 to $+0.50 \mathrm{D}$ and $\mathrm{AL}$ from 22.11 to $29.45 \mathrm{~mm}$. We believe that such a wide and consecutive range of refractive error would be more scientific to demonstrate the correlation between macular vascular parameters and ocular factors. Investigation of such correlation may reflect processes fundamental to the pathophysiologic characteristics of myopia $[9,37]$ and help develop the retinal vessel measurements as biomarkers for future vascular disease risk assessment with ocular elongation [34].

FA has been widely used to investigate retinovascular disorders. However, FA cannot dissociate the different networks that form the complex retinal vascularization. Moreover, it cannot be performed frequently due to its invasive nature. OCTA can visualize retinal capillaries by localizing the abnormalities to either the superficial or deep layer [13] and has the safety of not having to use an injected dye. However, in our study, between before and after magnification correction, there were significant differences of macular vasculature measurement with varying degrees of myopia. Moreover, correction for ocular magnification caused a reversal of relationship between the FAZ area and $\mathrm{AL}$ and $\mathrm{SE}$, and a more significant correlation between VD and $\mathrm{AL}$ and SE. Due to the longer AL, for a given visual angle, the scan area will be larger in a myopic retina than in an emmetropic retina, thus leading to a smaller measurement than the actual size [21]. Such discrepancy may interrupt the clinical use of this biomarker and lead to a confusing conclusion. Because the OCTA software cannot eliminate the effect of magnification, this study selected the modified AL method derived from Bennet because it was reported to make fewer assumptions about the optical dimensions of the eye and was proven to be as accurate as more detailed calculations using additional ocular biometric parameters [19, 38, 39].

This study has several limitations, including the limited sample in the emmetropia group. The cross-sectional design limited the variation of the macular vasculature parameters with myopic progression. Future studies will be composed of a large cohort and will use a longitudinal approach to reassess the predictive power of the index by OCTA in myopic complications. Owing to the intrinsic property of OCT, another limitation is that lower levels in the tridimensional data set may have blood vessels that in fact have the pattern of overlying vessels, which is referred to as a projection artefact [40]. Accordingly, the superficial capillary plexus may superimpose on the deep layer in part of OCTA images. In this study, by visual inspection, we excluded images of the deep capillary plexus with significant projections of the large vessels in the superficial layer. Moreover, transverse motions from fixation change in high myopia were another cause of artefacts in OCTA images. With the motion correction technology in the RTVue system, the quality of images was apparently improved [41]. Further, we excluded the cases with significant motion artefacts or doubling of vessels. However, it was still difficult to avoid all artefacts completely, which may lead to some confounding effects on the analysis of retinal vasculature in myopia. Future technologies may compensate for the projection artefact and correct the scans to deliver more precise results on the analysis of the retinal vascular system.

In summary, we demonstrated an increased FAZ and decreased vessel densities in the deep and superior vascular layers correlated with AL elongation. Among all the parameters of microvascular morphology, the VD in the deep vascular layer was most associated with capillary loss in high myopia, suggesting its use as an early indicator of macular complications related to high myopia. Measurements of VD based on OCTA images may provide additional information regarding the progression of pathologies underlying high myopic eyes.

\section{Summary}

\section{What was known before}

- An increased FAZ and decreased VD in both retinal layers were correlated with $\mathrm{AL}$ elongation. The decreasing deep perifoveal VD was most associated with high myopia.

\section{What this study adds}

- Measurements of OCTA images may provide additional information regarding the progression of pathologies in high myopia.

Funding This study was supported by research grants from the Medical and Health Project of Zhejiang Province (2016ZDA016 
to LS), the National Major Equipment Program of China (2012YQ12008004 to FL), the National Nature Science Foundation of China (Grant No. 81170869 and Grant No. 81570880 to FL and Grant No. 81400441 to LS), and the Public Service Program of Wenzhou Science and Technology Bureau (Y20160450 to DC and Y20160151 to QC).

\section{Compliance with ethical standards}

Conflict of interest The authors declare that they have no conflict of interest.

Publisher's note Springer Nature remains neutral with regard to jurisdictional claims in published maps and institutional affiliations.

\section{References}

1. Flitcroft DI. The complex interactions of retinal, optical and environmental factors in myopia aetiology. Prog Retin Eye Res. 2012;31:622-60.

2. Xu L, Li Y, Wang S, Wang Y, Wang Y, Jonas JB. Characteristics of highly myopic eyes: the Beijing eye study. Ophthalmology. 2007;114:121-6.

3. Benavente-Perez A, Hosking SL, Logan NS, Broadway DC. Ocular blood flow measurements in healthy human myopic eyes. Graefes Arch Clin Exp Ophthalmol. 2010;248:1587-94.

4. Kaiser HJ, Schoetzau A, Stumpfig D, Flammer J. Blood-flow velocities of the extraocular vessels in patients with high-tension and normal-tension primary open-angle glaucoma. Am J Ophthalmol. 1997;123:320-7.

5. Kaneko Y, Moriyama M, Hirahara S, Ogura Y, Ohno-Matsui K. Areas of nonperfusion in peripheral retina of eyes with pathologic myopia detected by ultra-widefield fluorescein angiography. Investig Ophthalmol Vis Sci. 2014;55:1432-9.

6. Shimada N, Ohno-Matsui K, Harino S, Yoshida T, Yasuzumi K, Kojima A, et al. Reduction of retinal blood flow in high myopia. Graefes Arch Clin Exp Ophthalmol. 2004;242:284-8.

7. La Spina C, Corvi F, Bandello F, Querques G. Static characteristics and dynamic functionality of retinal vessels in longer eyes with or without pathologic myopia. Graefes Arch Clin Exp Ophthalmol. 2016;254:827-34.

8. Li H, Mitchell P, Rochtchina E, Burlutsky G, Wong TY, Wang JJ. Retinal vessel caliber and myopic retinopathy: the blue mountains eye study. Ophthalmic Epidemiol. 2011;18:275-80.

9. Cheung N, Tikellis G, Saw SM, Amirul Islam FM, Mitchell P, Wang JJ, et al. Relationship of axial length and retinal vascular caliber in children. Am J Ophthalmol. 2007;144:658-62.

10. Dubis AM, Hansen BR, Cooper RF, Beringer J, Dubra A, Carroll J. Relationship between the foveal avascular zone and foveal pit morphology. Investig Ophthalmol Vis Sci. 2012;53:1628-36.

11. Tan CS, Lim LW, Chow VS, Chay IW, Tan S, Cheong KX, et al. Optical coherence tomography angiography evaluation of the parafoveal vasculature and its relationship with ocular factors. Investig Ophthalmol Vis Sci. 2016;57:OCT224-34.

12. Jin NY, Stjernschantz J. Regional blood flow in the myopic chick eye during and after form deprivation: a study with radioactivelylabelled microspheres. Exp Eye Res. 2000;71:233-8.

13. Spaide RF, Klancnik JM Jr., Cooney MJ. Retinal vascular layers imaged by fluorescein angiography and optical coherence tomography angiography. JAMA Ophthalmol. 2015;133:45-50.

14. Savastano MC, Lumbroso B, Rispoli M. In vivo characterization of retinal vascularization morphology using optical coherence tomography angiography. Retina. 2015;35:2196-203.
15. Shahlaee A, Pefkianaki M, Hsu J, Ho AC. Measurement of foveal avascular zone dimensions and its reliability in healthy eyes using optical coherence tomography angiography. Am J Ophthalmol. 2016;161:50-5 e1.

16. Yu J, Jiang $\mathrm{C}$, Wang $\mathrm{X}$, Zhu L, Gu R, Xu H, et al. Macular perfusion in healthy Chinese: an optical coherence tomography angiogram study. Investig Ophthalmol Vis Sci. 2015;56:3212-7.

17. Sunhee K, Youngjun K, Deukhee L, Sehyung P. Active contour segmentation using level set function with enhanced image from prior intensity. Conf Proc IEEE Eng Med Biol Soc. 2015;2015:3069-72.

18. Huang D, Chopra V, Lu AT, Tan O, Francis B, Varma R, et al. Does optic nerve head size variation affect circumpapillary retinal nerve fiber layer thickness measurement by optical coherence tomography? Investig Ophthalmol Vis Sci. 2012;53:4990-7.

19. Kang SH, Hong SW, Im SK, Lee SH, Ahn MD. Effect of myopia on the thickness of the retinal nerve fiber layer measured by Cirrus HD optical coherence tomography. Investig Ophthalmol Vis Sci. 2010;51:4075-83.

20. Bengtsson B, Krakau CE. Correction of optic disc measurements on fundus photographs. Graefes Arch Clin Exp Ophthalmol. 1992;230:24-8.

21. Bennett AG, Rudnicka AR, Edgar DF. Improvements on Littmann's method of determining the size of retinal features by fundus photography. Graefes Arch Clin Exp Ophthalmol. 1994;232:361-7.

22. Liu X, Shen M, Yuan Y, Huang S, Zhu D, Ma Q, et al. Macular thickness profiles of intraretinal layers in myopia evaluated by ultrahigh-resolution optical coherence tomography. Am J Ophthalmol. 2015;160:53-61 e2.

23. Dimitrova G, Tamaki Y, Kato S, Nagahara M. Retrobulbar circulation in myopic patients with or without myopic choroidal neovascularisation. Br J Ophthalmol. 2002;86:771-3.

24. Man REK, Lamoureux EL, Taouk Y, Xie J, Sasongko MB, Best WJ, et al. Axial length, retinal function, and oxygen consumption: a potential mechanism for a lower risk of diabetic retinopathy in longer eyes. Investig Ophthalmol Vis Sci. 2013;54: 7691-8.

25. Yang Y, Wang J, Jiang H, Yang X, Feng L, Hu L, et al. Retinal microvasculature alteration in high myopia. Investig Ophthalmol Vis Sci. 2016;57:6020-30.

26. Li M, Yang Y, Jiang H, Gregori G, Roisman L, Zheng F, et al. Retinal microvascular network and microcirculation assessments in high myopia. Am J Ophthalmol. 2017;174:56-67.

27. Al-Sheikh M, Phasukkijwatana N, Dolz-Marco R, Rahimi M, Iafe NA, Freund KB, et al. Quantitative OCT angiography of the retinal microvasculature and the choriocapillaris in myopic eyes. Investig Ophthalmol Vis Sci. 2017;58:2063-9.

28. Scarinci F, Nesper PL, Fawzi AA. Deep retinal capillary nonperfusion is associated with photoreceptor disruption in diabetic macular ischemia. Am J Ophthalmol. 2016;168:129-38.

29. Yi J, Liu WZ, Chen SY, Backman V, Sheibani N, Sorenson CM, et al. Visible light optical coherence tomography measures retinal oxygen metabolic response to systemic oxygenation. Light-Sci Appl. 2015;4:e334.

30. Lam DS, Leung KS, Mohamed S, Chan WM, Palanivelu MS, Cheung CY, et al. Regional variations in the relationship between macular thickness measurements and myopia. Investig Ophthalmol Vis Sci. 2007;48:376-82.

31. Wakitani Y, Sasoh M, Sugimoto M, Ito Y, Ido M, Uji Y. Macular thickness measurements in healthy subjects with different axial lengths using optical coherence tomography. Retina. 2003;23:177-82.

32. Mayama C, Suzuki Y, Araie M, Ishida K, Akira T, Yamamoto T, et al. Myopia and advanced-stage open-angle glaucoma. Ophthalmology. 2002;109:2072-7. 
33. Nemeth J, Michelson G, Harazny J. Retinal microcirculation correlates with ocular wall thickness, axial eye length, and refraction in glaucoma patients. J Glaucoma. 2001;10:390-5.

34. Gopinath B, Wang JJ, Kifley A, Tan AG, Wong TY, Mitchell P. The association between ocular biometry and retinal vascular caliber is comparable from early childhood to adolescence. Investig Ophthalmol Vis Sci. 2013;54:1501-8.

35. Patton N, Maini R, MacGillivary T, Aslam TM, Deary IJ, Dhillon B. Effect of axial length on retinal vascular network geometry. Am J Ophthalmol. 2005;140:648-53.

36. Lim LS, Cheung CYL, Lin XY, Mitchell P, Wong TY, Mei-Saw $S$. Influence of refractive error and axial length on retinal vessel geometric characteristics. Investig Ophthalmol Vis Sci. 2011;52:669-78.

37. Wong TY, Knudtson MD, Klein R, Klein BEK, Meuer SM, Hubbard LD. Computer-assisted measurement of retinal vessel diameters in the beaver dam eye study - methodology, correlation between eyes, and effect of refractive errors. Ophthalmology. 2004;111:1183-90.

38. Garway-Heath DF, Rudnicka AR, Lowe T, Foster PJ, Fitzke FW, Hitchings RA. Measurement of optic disc size: equivalence of methods to correct for ocular magnification. Br J Ophthalmol. 1998;82:643-9.

39. Nowroozizadeh S, Cirineo N, Amini N, Knipping S, Chang T, Chou $\mathrm{T}$, et al. Influence of correction of ocular magnification on spectral-domain OCT retinal nerve fiber layer measurement variability and performance. Investig Ophthalmol Vis Sci. 2014;55:3439-46.

40. Spaide RF, Fujimoto JG, Waheed NK. Image artifacts in optical coherence tomography angiography. Retina. 2015;35:2163-80.

41. Kraus MF, Potsaid B, Mayer MA, Bock R, Baumann B, Liu JJ, et al. Motion correction in optical coherence tomography volumes on a per A-scan basis using orthogonal scan patterns. Biomed Opt Express. 2012;3:1182-99. 